\title{
Self-Organization of Value and Demand
}

\author{
R. Donangelo ${ }^{a}$ and K. Sneppen ${ }^{b}$ \\ ${ }^{a}$ Instituto de Física, Universidade Federal do Rio de Janeiro, \\ C.P. $68528,21945-970$ Rio de Janeiro, Brazil \\ ${ }^{b}$ Nordita, Blegdamsvej 17, 2100 Copenhagen, Denmark
}

\begin{abstract}
We study the dynamics of exchange value in a system composed of many interacting agents. The simple model we propose exhibits cooperative emergence and collapse of global value for individual goods. We demonstrate that the demand that drives the value exhibits non-Gaussian "fat tails" and typical fluctuations which grow with time interval as $\Delta t^{H}$, with $H \sim 0.7$.

PACS numbers: 02.50, 05.10.-a, 05.40.-a, 05.45.-a, 05.65.+b, 05.70.Ln, 87.23.Ge, 89.90. $+\mathrm{n}$
\end{abstract}

The self organizational patterns of large non-equilibrium systems are of large current interest. One aspect of such systems is their tendency to display cooperative behavior, evidenced, for example, in the form of the occasional coordinated activity throughout the system [0 [3]. Economic systems provide examples of such cooperative behavior, that indeed show some of the characteristics of self organizing systems. More specifically, agents reach a collective agreement about what should be considered as valuable, and then use it as a common trade object (money) [4. Furthermore, the fluctuation of relative value with time $t$ displays anomalous Hurst exponents (typical $\Delta D \propto \Delta t^{H}$ [5] with $H>1 / 2$ ) [6] [] and non-Gaussian statistics. The latter fact was first noticed by Mandelbrot [8.9] and later quantified in the observation that the succession of daily, weekly and monthly distributions of exchange values possibly converges towards a Gaussian [10,11].

Recently a number of theoretical approaches have been developed to deal either with the 
emergence of money as a cooperative phenomenon, or with the non-Gaussian fluctuations associated to it. In particular the work of Yasutomi [12 deals with the stochastic nature of emergence of certain goods as means of common exchange between all the agents, i.e. how they become money. Yasutomi makes the observation that if agents tends to accept what others accept, then trade is facilitated. Therefore, emergence of money becomes related to history dependent processes, which, as discussed by B. Arthur [13 tend to lock the market into certain trade patterns. This, however does not explain how one popular product may be replaced by another, and thus result in an open and ever fluctuating system. Yasutomi suggestion to solve this problem is to include both trade costs and an evolving threshold for transactions. In this way he obtains a bi-stable system where money emerges and collapses quasiperiodically. Dealing with the nature of fluctuations alone, Bak, Paczuski and Shubik suggested a model with only one product, and concluded that non-Gaussian fluctuations could be associated with the crowding obtained when agents imitate each other's prize assessments [14]. They further obtained the anomalous Hurst exponents $H=0.65$ in a scenario where local fluctuations are amplified by the global activity level. Levy, Levy and Solomon [15] emphasize the effect of heterogeneous expectations for the traders in a stockbond market. However, their specific model develops an unrealistic periodicity for large systems [16]. As we will see, our model self organizes heterogenity in a market where each agent has its own limited history. Furthermore, it predicts the emergence of non-periodic but persistent fluctuations in a market where many products compete for attention.

In the present work we suggest a simple model that suggests that emergence of "money" and its anomalous fluctuation in value are two sides of the same cooperative phenomena. We suggest that value emerges through agents which make simple decisions based on their individual memory of earlier encounters with other agents. The agents are not assumed to be smart, the only trade that occurs is a one-good-for-one-good trade, and agents basically act in order to keep stock of all products. The model leads to emergence and collapse of money, it exhibits non-Gaussian statistics and also displays long time fluctuations quantified by anomalous Hurst exponents. 
The model we propose is as follows. We consider $N_{a g}$ agents and $N_{p r}$ different products. Initially we give $N_{\text {unit }}$ units of the products to each agent. The number $N_{\text {unit }}$ is fixed, but the products are chosen at random, so the individuals are not in exactly the same situation.

At each timestep we select two random agents and let them attempt to perform a trade between them. The trade starts by comparing the list of goods that each agent lacks and therefore would like to get from the other agent in exchange for goods it has in stock.

We first consider the simple barter exchange procedure: when each of the agents has products that the other needs, then one of these products, chosen at random, is exchanged.

In case such a barter exchange is not possible we consider the "money" exchange procedure: one or both of the agents accept goods which they do not lack, but consider useful for future exchanges.

In order to determine the usefulness of a product, each agent $i$ keeps a record of the last requests for goods it received in encounters with other agents. This memory is finite, having a length of $N_{\text {mem }}$ positions, each of which registers a product that was requested. As the memory gets filled, the record of old transactions is lost. Agents accept products they already have in stock with a probability based on its memory record. The chance of accepting such a good $j$ is taken to be proportional to the number of times $T_{i j}$ that good $j$ appears on the memory list of agent $i$ :

$$
p_{i j}=\frac{T_{i j}}{N_{m e m}}
$$

where we have used the fact that $\sum_{j} T_{i j}=N_{m e m}$. These two exchange mechanisms define our model.

In order to understand the dynamics of this model we first show, in Fig. 1, the time evolution of the number of different kinds of trades. The case depicted corresponds to $N_{a g}=200$ agents trading $N_{p r}=200$ types of products between them. The memory list of each agent was chosen to be $N_{m e m}=400$ items long and the total "richness" of the economy 
was fixed by setting the numbers of products possessed by each agent to $N_{\text {unit }}=400$. This may be considered a typical set of parameters, and the behavior is similar for a wide range of number of goods, memory sizes and richness values, as long as memories are not too short and richness not too high or too small. If the economy is too rich, barter exchange disappears completely as all agents will always have all types of goods. On the other hand, if the economy is very poor, barter exchange dominates completely and "money" exchange does not emerge. Besides, in order for money to appear at all, the memory of each agent must not be much shorter than the number of different products, $N_{p r}$, (however, it can be much smaller than $N_{a g}$ ).

Fig. 1 shows that after a relatively short equilibration time the ratio of barter to money transactions saturates. The fact that money transactions dominate means that the agents have already distributed their holdings in an efficient way, thus allowing money exchange to become the dominating mode of transaction. We also notice that the sum of the two types of exchanges is less than 100\%, meaning that approximately $40 \%$ of encounters between agents did not lead to trades, for this set of parameters.

The interesting feature of our model is however not the relative number of these two types of transactions, but the the fact that some products become valuable as means of exchange. In order to quantify this we define the monetary value of a good $j$ as the number of agents $M=M(j)$ that consider this good to be the easiest to trade. In other words, each agent $i$ indicates the good $j$ with highest $p_{i j}$.

In Fig. 2a,b we show the value of two particular goods as function of time, which here we measure as number of encounters between agents. Notice that the time scale for the evolution is a factor 100 larger than that of Fig. 1. This shows that, although the number of money and barter exchanges has equilibrated almost instantaneously in the timescale of Fig. 2, the evolution of the value of the goods displays an interesting dynamics on this larger timescale. In Fig. 2c we have plotted the number of agents that accept the most desirable good. From comparison with Figs. 2a and 2b, we notice that the good in Fig. 2a becomes the most accepted in terms of exchange early in the evolution of the system, and remains 
in that condition until more than 900000 encounters have taken place, in this particular history. At this point, the good from Fig. $2 \mathrm{~b}$ takes over, for a briefer period, until time 1200000. There is a couple of additional crossovers between these two products, but at the end another product arises and takes over as being the most popular.

We stress that an important feature of the model is that often, and over long periods, one particular good is considered valuable by a majority of the agents. This appears without any a priori property of this product; the value of the good develops through a cooperative agreement between the agents about what is valuable.

How is this agreement reached? In our model the agents only transfer information about what they need to replenish their stocks. Thus the common concept of value emerges when many agents need a good, e.g. because it is concentrated by some of the agents. This accumulation is possible in our simple model because an agent accepts goods even when having them in sufficient stock, if it feels they will be useful for future transactions. This property in itself makes a desired product to circulate, and possibly concentrate, more than other goods, thereby making it more needed across the system.

However goods in high demand also collapse, as it was seen at time $\approx 800000$ in Fig. 2a. This is because, due to the large number of money transactions, the most valuable good may, through random fluctuations, become better distributed than some other good, which then replaces it as the system's money.

We now try to quantify these fluctuations. Fig. 3a displays the changes in the value of $M$, which time evolution was seen in Fig. 2c, for various time intervals $\Delta t$. The figure shows that, for sufficiently large values of $\Delta t$, these fluctuations have exponential tails. This is in contrast to the normal Gaussian behavior expected for value assigned by independent agents. The fluctuations obtained for the different values of $\Delta t$ could not be made to collapse into one curve. This signals that the short and long time statistics of $M$ cannot be described by the same Hurst exponent.

In order to examine the statistics of the underlying demand of goods we show, in Fig.3b, the fluctuations in the number of times $D$ that a given product $j$ appears in the memory of 
the system,

$$
D(j)=\sum_{i} T_{i j}
$$

where the sum runs over all agents $i$ in the system. In Fig. 3b we show a data collapse of fluctuations in $D$, i.e. $P(\Delta D) \rightarrow \Delta t^{H} \cdot P\left(\frac{\Delta D}{\Delta t^{H}}\right)$ for 3 different values of $\Delta t$. We notice that the curves collapse with Hurst exponent $H=0.70$, an observation that we confirmed by finding numerically that $\left(\left\langle(D(t+\Delta t)-D(t))^{2}\right\rangle\right)^{\frac{1}{2}} \propto \Delta t^{0.68 \pm 0.02}$, over more than three orders of magnitude. The fact that $H>0.5$ indicates that fluctuations in demand exhibit persistency, i.e. that trends are amplified by a self organized cooperative feedback in our model. This relatively large anomalous Hurst exponent requires that there is a sizeable number of different products. We have found that persistence exists for nearly all system sizes. The value of the Hurst exponent was found to 0.6 for a size 10 system whereas it was found to be 0.7 for system size 100 and 1000. For size dependence we mean here that we scale equally the parameters of the model: $N_{a g}, N_{p r}, N_{m e m}, N_{\text {unit }}$. We have also checked that a change in $N_{a g}$ alone from 100 to 1000 also leads to a Hurst exponent of 0.7 . Only in the case of $N_{p r}=2$ we found $H=0.5$, and this for any value of the other parameters.

We further notice, from Fig. 3b, that the fluctuations exhibit fat tails. In particular, the short time scale statistics tend to have exponential tails whereas the long timescale fluctuations are more Gaussian-like. The difference of Hurst scaling between value $M$ and demand $D$ reflects the fact that changes in the most wanted product are faster, but less persistent than structural changes in the composition of demand.

We have examined variations of the above model, as, for example, including reluctance for the agents to trade away goods they consider valuable, i.e. with high $p_{i j}$. Also we have considered the possibility that agents include in $T_{i}$ only trades that were effectively performed, and not just requests. Finally, we have tested the case where the $T_{i}$ lists also include requests for "money trades", arising from what other agents consider valuable. All these cases change the information exchanged between agents about what is valuable. However, the qualitative behavior of our model was in all cases similar, indicating its robustness. 
It is tempting to compare the emergence of value in the above model with the emergence of monetary systems in the real world. A beautiful historical example of the collapse of one currency and the appearance of a new one in a XVII century chinese town is given in Yasutomi's work [12]. More familiar to the reader may be similar events in the market for tulips in the Netherlands, or the market for gold in more recent times. In all these cases the acceptability of the good or currency may drop dramatically, without any deeper reason than the fact that nobody considers them valuable any more. More quantifiable are the values of currencies, where now, due to events that have taken place in this century, the U.S. Dollar has become globally accepted. In that regards one should mention that fluctuations in monetary value seem to exhibit fat tails [7,8], and long time fluctuations that can be characterized by a Hurst exponent $H \approx 0.55$ [7]. All these features are consistent with our simple scenario. We stress that our model is schematic and does not include any development of strategy by the agents, strategies which would naturally influence the emergence of cooperativity [17,18].

Furthermore, the present version of the model does not include effects related to production or consumption of goods. We have verified that the main result of including these processes, is that products that are easily produced never become valuable. Thus, if all goods are produced and consumed at a high rate, no common "money" will emerge. On the other hand, if products are produced at a low rate the results presented above remain valid, indicating again the robustness of the present model. In fact, the addition of a slow production and consumption of goods allows the system to settle into a statistical stationary state. This avoids products from falling, one by one, into the absorbing state consisting of having the product present in the stock of all agents, and, consequently, being gradually removed from the memories. As soon as such a product is removed from all memories, it is effectively not anymore present in the economy. The effect of the decay mentioned above is negligible in the time scale of the calculations presented in this work.

Finally we would like to emphasize that our work purports to model behavior in a social setting where information exchange is a key feature. The fact that some forms of information 
exchange lead to self-organization has already been proposed by e.g. Bonabeau et al. [20]. There sociological hierarchies emerge through comparisons of a dominance index assigned to each individual. Our model is more adapted for market behavior, in particular because it also considers the exchange of goods, and not only of information. By emphasizing the interplay between exchange of products and information, we have constructed a minimalistic market model. However, since we explicitly require that our agents do not develop different strategies, and that all the products can be exchanged only in a one-to-one manner, our model may not give answers to some questions that may be adressed in more detailed simulation models e.g. employing minority games [17], or the more elaborate models of strategy and investments programs also existing in the economic literature, e.g. the one by Kim and Markowitz [19].

In summary, we have constructed a simple model for the cooperative concept of money. The model suggests that money emerges and collapses as a simple consequence of trade of goods between interacting agents with memory. The concept of value arises from the probability that a local dynamical pattern (the need for certain products by individual agents) results in a global one (the general acceptability of products for exchange). An important consequence, possibly valid for the dynamics of other large systems, is that a tendency to draw information from previously encountered patterns may result in a dynamic behavior that exhibits non-Gaussian fluctuations and persistency, evidenced by anomalous Hurst exponents.

We thank Maya Paczuski and Dietrich Stauffer for constructive comments to the manuscript. R.D. thanks Nordita for its hospitality and financial support during the time that this work was performed. 


\section{REFERENCES}

[1] P. Bak, C. Tang and K. Wiesenfeld, Phys. Rev. Lett 59 (1987) 381.

[2] K. Sneppen, P. Bak, H. Flyvbjerg and M.H. Jensen, Proceedings National Academy of Science, USA, Vol. 92 (1995) 5209.

[3] S. Stange, Z. Zanette, A. Mikhailov and B. Hess, Biophysical Chemistry 72 (1998) 73.

[4] C. Menger, Principles of Economics (1871), translated by J. Dingwall and B.F. Hoselits (Glencoe,I 11., 1950).

[5] H.E. Hurst, Tr. of the American Society of Civil Engineers, 116 (1951) 770.

[6] R.N. Mantegna, Physica A 179 (1991) 232.

[7] C.J.G. Evertz, Fractals 3 (1995) 609.

[8] B. Mandelbrot, Journal of Business of the University of Chicago 36 (1963) 307.

[9] B. Mandelbrot, "the Journal of Business of the University of Chicago" 40 (1967) 393.

[10] V. Akgiray and G.G. Booth, J. Business Econ. Statistics 6 (1988) 51.

[11] S. Ghashghaie, W. Breymann, J. Peinke, P. Talkner and Y. Dodge, Nature 381 (1996) 767.

[12] A. Yasutomi Physica D 82 (1995) 180.

[13] W.B. Arthur, "Increasing Returns and Path Dependence in the Economy", The University of Michigan Press, (1997).

[14] P. Bak, M. Paczuski and M. Shubik, Physica A 246 (1997) 430.

[15] M. Levy, H. Levy and S. Solomon, J. Phys. I France 5 (1995) 1087.

[16] . T. Hellthaler, Int. J. Mod. Phys. C6 (1995) 845; R. Kohl, Int. J. Mod. Phys. C8 (1997) 1309. 
[17] D. Challet and Y.-C. Zhang, Physica A 246 (1997) 407; Y.-C. Zhang, Europhys. News 29 (1998) 51.

[18] M. Paczuski and K. Bassler, cond-mat/9905082.

[19] G.R. Kim and H. M. Markowitz, J. Portfolio Management 16 (1989) 45.

[20] E. Bonabeau, G. Theraulaz and J-L. Deneubourg, Physica A217 (1995) 373.

\section{Figure Captions}

- Fig. 1. Probability, measured as a percentage of the encounters between agents that result in barter (full line) or money exchanges (dashed line), as defined in the text.

- Fig. 2. Number of agents $M$ that consider a particular product as the most valuable ( $a$ and $b$ ), and the maximum number of agents that, at a given time, consider the same product as the most valuable (c).

- Fig. 3. (a) Probability of having changes in the value $M$ as a function of their size, for the three different time steps $\Delta t=200000$ (full line), $\Delta t=20000$ (long dashed line), and $\Delta t=2000$ (short dashed line). (b) Probability of having changes in the demand $D$. The demand is here measured in units of $\Delta D / \Delta t^{H}$, where $\Delta t$ takes the values 200, 2000, and 20000 (three thin lines), and we took for the Hurst exponent the value $H=0.7$. The thick line depicts the normal probability distribution having the same mean and standard deviation as that for $\Delta t=200$. The peaks near 0 observed in the other distributions are attributed to border effects, arising from transitions near $D=0$. 